\title{
POSITIVE DEFINITE MATRICES AND CATALAN NUMBERS
}

\author{
FRANK THOMSON LEIGHTON AND MORRIS NEWMAN ${ }^{1}$
}

\begin{abstract}
It is shown that the number of $\boldsymbol{n} \times \boldsymbol{n}$ integral triple diagonal matrices which are unimodular, positive definite and whose sub and super diagonal elements are all one, is the Catalan number $\left(\begin{array}{c}2 n \\ n\end{array}\right) /(n+1)$. More generally, it is shown that if $A$ is a fixed integral symmetric matrix and $d$ is a fixed positive integer, then there are only finitely many integral diagonal matrices $D$ such that $A+D$ is positive definite and $\operatorname{det}(A+D)=d$.
\end{abstract}

1. Introduction. It is well known and easy to prove that any integral symmetric matrix $A$ is integrally congruent to a triple diagonal matrix (see [1, p. 60], for example). Joan Birman asked the second author whether it is always possible to make all the elements on the sub and super diagonals one, provided that $\operatorname{det}(A)=$ 1. It is readily shown that this is not possible. For example, if $A$ is positive definite and is not integrally congruent to the identity, then $A$ cannot be integrally congruent to a matrix of the type desired, since all such matrices are integrally congruent to the identity (this fact is proved in Theorem 2). The question raised by Professor Birman suggested that it would be of interest to study the class of $n \times n$ integral triple diagonal matrices which are unimodular, positive definite and whose sub and super diagonal elements are all one. We will show that this class is finite and that its cardinality is just the Catalan number $\left(\begin{array}{c}2 n \\ n\end{array}\right) /(n+1)$. This supplies a rather surprising new interpretation for the Catalan numbers. In addition, we will prove a general theorem of this type for arbitrary integral symmetric matrices.

If positive definiteness is not required, then the class of matrices under consideration need not be finite. For example, each of the matrices

$$
\left[\begin{array}{rrr}
-1 & 1 & 0 \\
1 & -1 & 1 \\
0 & 1 & a
\end{array}\right], \quad\left[\begin{array}{rcc}
-a & 1 & 0 \\
1 & 0 & 1 \\
0 & 1 & a-1
\end{array}\right]
$$

is unimodular for all integral values of $a$.

2. A general result. We first prove a result for arbitrary integral symmetric matrices. In the following lemma, $E_{i j}$ denotes the matrix with a one in position $(i, j)$ and zeros elsewhere.

Received by the editors March 20, 1978 and, in revised form, May 1, 1979; presented to the Society, April 23, 1977.

AMS (MOS) subject classifications (1970). Primary 15A36, 05 A15.

Key words and phrases. Catalan number, congruence, determinant, integer matrix, positive definite matrix, triple diagonal matrix, unimodular matrix.

'The work of this author was supported by NSF grant MCS76-8293. 
LEMMA 1. If $B=\left[b_{i j}\right]$ is a symmetric positive definite matrix, $c$ is a positive number, and $B^{\prime}=B+c E_{j j}$ for some value of $j$, then $\operatorname{det}\left(B^{\prime}\right)>\operatorname{det}(B)$.

Proof. If we compute $\operatorname{det}\left(B^{\prime}\right)$ by minors of the $j$ th row, we find that $\operatorname{det}\left(B^{\prime}\right)=$ $\operatorname{det}(B)+c d_{j}$ where $d_{j}$ is the cofactor of $b_{j j}$, and hence positive, since $B$ is positive definite.

We now prove

Theorem 1. If $A$ is a fixed $n \times n$ integral symmetric matrix and $d$ is a fixed positive integer, then the number of integral diagonal matrices $D$ such that $A+D$ is positive definite and $\operatorname{det}(A+D)=d$ is finite.

Proof. We may assume without loss of generality that $A$ has zeros on the diagonal. Then if $A+D$ is positive definite, $D$ must have positive diagonal elements. Let $\mathscr{D}$ be the class of all $D$ such that $A+D$ is positive definite and $\operatorname{det}(A+D)=d$. From repeated application of Lemma 1 , it is clear that if $F=\left[f_{i j}\right]$ and $G=\left[g_{i j}\right]$ are different elements of $\mathscr{D}$, then there exist $k$ and $m$ such that $f_{k k}>g_{k k}$ and $f_{m m}<g_{m m}$. Otherwise it would not be possible for $\operatorname{det}(A+F)$ to equal $\operatorname{det}(A+G)$. Thus $\mathscr{D}$ forms an antichain (order-independent set) in $\mathbf{N}^{n}$. Since all antichains in $\mathbf{N}^{n}$ are known to be finite [2], there can be only a finite number of elements in $\mathscr{D}$.

It is clear that generalizations of this result are possible (the diagonal matrices $D$ may be replaced by symmetric positive definite matrices, for example) but we do not consider these here.

3. The basic framework. Define

$$
A=\operatorname{td}\left(a_{1}, a_{2}, \ldots, a_{n}\right)=\left[\begin{array}{ccccc}
a_{1} & 1 & & & \\
1 & a_{2} & 1 & & \\
& \cdots & & & \\
& & 1 & a_{n-1} & 1 \\
& & & 1 & a_{n}
\end{array}\right]
$$

Let $D_{k}$ be the determinant of the minor matrix of $A$ obtained by striking out the last $n-k$ rows and columns, and let $E_{k}$ be the determinant of the minor matrix of $A$ obtained by striking out the first $n-k$ rows and columns. In addition, define

$$
D_{-1}=0, \quad D_{0}=1, \quad E_{-1}=0, \quad E_{0}=1 .
$$

Then the $D_{k}$ 's and the $E_{k}$ 's satisfy the recurrences

$$
\begin{array}{ll}
D_{k}=a_{k} D_{k-1}-D_{k-2}, & 1<k<n, \\
E_{k}=a_{n-k+1} E_{k-1}-E_{k-2}, & 1<k<n .
\end{array}
$$

Furthermore, expanding $\operatorname{det}(A)$ by minors of the $k$ th row of $A$, we find that

$$
\begin{aligned}
\operatorname{det}(A) & =a_{k} D_{k-1} E_{n-k}-D_{k-2} E_{n-k}-D_{k-1} E_{n-k-1} \\
& =D_{k} E_{n-k}-D_{k-1} E_{n-k-1}, \quad 1<k<n .
\end{aligned}
$$

We are interested in the matrices $A$ given by (1) which are unimodular and positive definite. A necessary and sufficient condition for this to happen is that 
$a_{1}, a_{2}, \ldots, a_{n}$ be positive integers such that

$$
D_{k}>0, \quad 1 \leqslant k \leqslant n-1, \text { and } D_{n}=1 \text {. }
$$

The set of such matrices $A$ will be denoted by $S_{n}$, and will be the object of our study. We commence with some elementary lemmas.

LEMMA 2. If $A=\operatorname{td}\left(a_{1}, \ldots, a_{n}\right) \in S_{n}$, then $a_{k}=1$ for some $k, 1<k<n$.

Proof. Assume that $a_{i} \geqslant 2$ for $1 \leqslant i \leqslant n$. Then $D_{0}=1, D_{1}=a_{1}>2$, and $D_{k}=a_{k} D_{k-1}-D_{k-2} \geqslant 2 D_{k-1}-D_{k-2}$ for $2<k<n$. Thus $D_{k}-D_{k-1}>D_{k-1}$ $-D_{k-2}$ for $2 \leqslant k \leqslant n$ and $D_{k} \geqslant k+1$ for $1<k \leqslant n$. In particular, this means that $D_{n}>n+1>1$ for $n \geqslant 1$, which is a contradiction.

In the following, we write [1] $\dot{+}$ to denote the direct sum of the matrices [1] and $C$. In addition, we use the sequence notation $a_{k}, \ldots, a_{r}-1$ to denote the sequence $a_{k}, a_{k+1}, \ldots, a_{r-1}, a_{r}-1$ when $r>k$, the integer $a_{k}-1$ when $r=k$, and the empty sequence when $r<k$. Similar interpretations are understood for $a_{k}, \ldots, a_{r}+1$, for $a_{k}-1, \ldots, a_{r}$, and for $a_{k}+1, \ldots, a_{r}$. We are now ready to prove

LeMMA 3. Let $a_{1}, \ldots, a_{n}$ and $k$ be integers such that $a_{k}=1$ and $1<k<n$. Then $A=\operatorname{td}\left(a_{1}, \ldots, a_{n}\right)$ is integrally congruent to $[1]+\operatorname{td}\left(a_{1}, \ldots, a_{k-1}-1, a_{k+1}-\right.$ $\left.1, \ldots, a_{n}\right)$.

Proof. Perform the following congruence transformations on $A$. Subtract row $k$ from rows $k-1$ and $k+1$ (as far as present) and then do the same with the columns. Multiply the first $k-1$ rows and columns by -1 . Now permute the rows and columns of the matrix according to the permutation $(123 \cdots k)$. It is not difficult to check that the resulting matrix is $[1]+\operatorname{td}\left(a_{1}, \ldots, a_{k-1}-1, a_{k+1}-\right.$ $\left.1, \ldots, a_{n}\right)$.

As an important consequence of Lemma 3, we note without proof:

LeMmA 4. $\operatorname{td}\left(a_{1}, \ldots, a_{n}\right) \in S_{n}$ if and only if $\operatorname{td}\left(a_{1}, \ldots, a_{k-1}+1,1, a_{k}+\right.$ $\left.1, \ldots, a_{n}\right) \in S_{n+1}$ for every integer $k, 1<k<n+1$.

4. The class $S_{n}$. We are now prepared to prove the main results of this paper.

THEOREM 2. Every element of $S_{n}$ is integrally congruent to the identity.

Proof. The result is trivial for $n=1$. Suppose the theorem is true for $n-1$ where $n \geqslant 2$. Let $A$ be any element of $S_{n}$. From Lemmas 2, 3 and 4, we know that there is a matrix $B$ such that $A$ is integrally congruent to [1] $\dot{+} B$ and $B \in S_{n-1}$. By the induction hypothesis, $B$, and thus $A$, is congruent to the identity.

THEOREM 3. The set $S_{n}$ has cardinality $\left(\begin{array}{c}2 n \\ n\end{array}\right) /(n+1)$.

Proof. The proof consists of two parts. In the first, we show that for every $r$, $1<r<n$, any element of $S_{r-1}$ may be "composed" with any element of $S_{n-r}$ to determine in a unique fashion an element of $S_{n}$. In the second part of the proof, we show that, conversely, every element of $S_{n}$ is uniquely "decomposable" into an element of $S_{r-1}$ and an element of $S_{n-r}$ for exactly one value of $r, 1<r<n$. It 
will then immediately follow that

$$
\left|S_{n}\right|=\sum_{r=1}^{n}\left|S_{r-1}\right|\left|S_{n-r}\right|, \quad n>1, \quad\left|S_{0}\right|=1 .
$$

Since the recurrence relation (6) defines the Catalan numbers, we will thus have shown that $\left|S_{n}\right|=\left(\begin{array}{c}2 n \\ n\end{array}\right) /(n+1)$.

If $A=\operatorname{td}\left(a_{1}, \ldots, a_{n}\right) \in S_{n}$, then for each $r, 1 \leqslant r<n, a_{r}$ is uniquely determined by the other $a_{i}, 1<i \leqslant n, i \neq r$. This follows from formulas (2)-(4) and the fact that $\operatorname{det}(A)=1$. In fact,

$$
a_{r}=\left(1+D_{r-2} E_{n-r}+D_{r-1} E_{n-r-1}\right) / D_{r-1} E_{n-r} .
$$

Because of (7), we can also say that if $\operatorname{td}\left(a_{1}, \ldots, a_{r-1}\right) \in S_{r-1}$ and $\operatorname{td}\left(a_{r+1}, \ldots\right.$, $\left.a_{n}\right) \in S_{n-r}$ for any integers $a_{1}, \ldots, a_{r-1}, a_{r+1}, \ldots, a_{n}$, then there is a unique integer $a_{r}$ such that $\operatorname{td}\left(a_{1}, \ldots, a_{n}\right) \in S_{n}$. In fact, since $D_{r-1}=E_{n-r}=1, a_{r}$ is given by

$$
a_{r}=1+D_{r-2}+E_{n-r-1} \text {. }
$$

This completes the first part of the proof.

Now suppose that $\operatorname{td}\left(a_{1}, \ldots, a_{n}\right) \in S_{n}$. We say that $a_{r}$ is a breakpoint if $\operatorname{td}\left(a_{1}, \ldots, a_{r-1}\right) \in S_{r-1}$ and $\operatorname{td}\left(a_{r+1}, \ldots, a_{n}\right) \in S_{n-r}$. In the following, we will show that every element of $S_{n}$ possesses a breakpoint. This is trivially true for $n=1$. Assume that it is true for $n-1$ where $n \geqslant 2$. Let $A=\operatorname{td}\left(a_{1}, \ldots, a_{n}\right)$ be any element of $S_{n}$. We know by Lemmas 2 and 4 that there is a $k$ such that $1<k<n$, $a_{k}=1$ and $B=\operatorname{td}\left(a_{1}, \ldots, a_{k-1}-1, a_{k+1}-1, \ldots, a_{n}\right) \in S_{n-1}$. By the induction hypothesis, this element has a breakpoint which divides the sequence of diagonal elements into two subsequences of lengths $r-1$ and $n-r-1$, say, and which yield elements of $S_{r-1}$ and $S_{n-r-1}$. The subsequence that does not contain $a_{k-1}-1$ or $a_{k+1}-1$ is contained identically in the original sequence of diagonal elements $a_{1}, \ldots, a_{n}$. The other subsequence is empty or contains one or both of $a_{k-1}-1$ and $a_{k+1}-1$. In any case, we know by Lemma 4 that we can insert a 1 and add a 1 to the adjoining number(s) to get the complementary part of $a_{1}, \ldots, a_{n}$, and that this new sequence will be the sequence of diagonal elements of a member of $S_{r}$ or of $S_{n-r}$. Thus $A$ has a breakpoint, and the induction is complete.

We now show that the location of the breakpoint is unique. This is certainly true for $n=1$. Assume that it is true for $n-1$ where $n \geqslant 2$. Let $A=\operatorname{td}\left(a_{1}, \ldots, a_{n}\right) \in$ $S_{n}$, and suppose that $A$ has two breakpoints $a_{i}$ and $a_{j}, i \neq j$. By (8), we must have $a_{r}=1+D_{r-2}+E_{n-r-1}$ for $r=i, j$. It follows that if $n>1$, then $a_{i}>1$ and $a_{j}>1$. Thus there is a $k$ different from $i$ and $j$ such that $a_{k}=1$. But this means that $B=\operatorname{td}\left(a_{1}, \ldots, a_{k-1}-1, a_{k+1}-1, \ldots, a_{n}\right) \in S_{n-1}$. Thus $a_{i}$ and $a_{j}$ are also breakpoints of $B$, which contradicts the inductive hypothesis. Finally, formula (8) insures the uniqueness of the value of $a_{r}$ at the breakpoint. This completes the induction and establishes the uniqueness of the decomposition.

5. Additional results. In this section, we list some miscellaneous results, some of which have not been proved completely.

(9) If $\operatorname{td}\left(a_{1}, \ldots, a_{n}\right) \in S_{n}$ and $D_{0}, \ldots, D_{n}$ are as in formulas (2)-(5), then 
(i) $a_{i}<n$ for $1<i<n$,

(ii) $2 n-1<\sum_{i=1}^{n} a_{i}<3 n-3$,

(iii) $D_{k} / D_{k+1}<k+1$ for $0<k<n$,

(iv) $D_{k} / D_{k-1}<n-k+1$ for $0<k<n$.

(10) The number of elements of $S_{n}$ with trace $t$ is given by

$$
\left(\begin{array}{c}
t-n-1 \\
n-2
\end{array}\right) \frac{(3 n-t-1)(3 n-t-2)}{n-1} \text { if } 2 n-1<t<3 n-3
$$

0 otherwise.

(11) The number of elements of $S_{n}$ with exactly $i$ diagonal elements equal to one is given by

$$
\begin{aligned}
& \frac{2^{n-2 i+1}(n-1) !}{(n-2 i+1) ! i !(i-1) !} \text { if } 0<i<\left[\frac{n+3}{2}\right] \text {, } \\
& 0 \text { otherwise. }
\end{aligned}
$$

(9) is straightforward but (10) and (11) seem more difficult. We do not believe them to offer any fundamental problems however, and the reader is invited to supply proofs.

We are indebted to the referee for his careful reading of the paper and for his numerous valuable comments.

\section{REFERENCES}

1. Morris Newman, Integral matrices, Academic Press, New York, 1972.

2. Richard Stanley, Elementary problems and solutions problem E2546, Amer. Math. Monthly 83 (10) (1976), 813-814.

National Bureau of Standards, Operations Research Division, Washington, D.C. 20234

INSTITUTE FOR THE INTERDISCIPLINARY APPLICATIONS OF ALGEBRA AND COMBINATORICS, DEPARTMENT of Mathematics, University of California, SANta Barbara, California 93106 (Curtent address of Morris Newman)

Current address (F. T. Leighton): Applied Mathematics Department, Massachusetts Institute of Technology, Cambridge, Massachusetts 02139 